ARTICLE

Received 26 May 2015 | Accepted 3 Sep 2015 | Published 9 Oct 2015

DOI: 10.1038 /ncomms 9546

OPEN

\title{
Substrate specificity and transport mechanism of amino-acid transceptor Slimfast from Aedes aegypti
}

\author{
Dmitri Y. Boudko ${ }^{1}$, Hitoshi Tsujimoto ${ }^{2}$, Stacy D. Rodriguez ${ }^{2}$, Ella A. Meleshkevitch ${ }^{1}$, David P. Price ${ }^{3}$, Lisa L. Drake ${ }^{2}$ \\ \& Immo A. Hansen 2,3,4
}

Anautogenous mosquitoes depend on vertebrate blood as nutrient source for their eggs. A highly efficient set of membrane transporters mediates the massive movement of nutrient amino acids between mosquito tissues after a blood meal. Here we report the characterization of the amino-acid transporter Slimfast (Slif) from the yellow-fever mosquito Aedes aegypti using codon-optimized heterologous expression. Slif is a well-known component of the target-of-rapamycin signalling pathway and fat body nutrient sensor, but its substrate specificity and transport mechanism were unknown. We found that Slif transports essential cationic and neutral amino acids with preference for arginine. It has an unusual dual-affinity mechanism with only the high affinity being $\mathrm{Na}^{+}$dependent. Tissue-specific expression and blood meal-dependent regulation of Slif are consistent with conveyance of essential amino acids from gut to fat body. Slif represents a novel transport system and type of transceptor for sensing and transporting essential amino acids during mosquito reproduction.

\footnotetext{
${ }^{1}$ Department of Physiology and Biophysics, Rosalind Franklin University, North Chicago, Illinois 60064, USA. ${ }^{2}$ Department of Biology, New Mexico State University, Las Cruces, New Mexico 88003, USA. ${ }^{3}$ Molecular Biology Program, New Mexico State University, Las Cruces, New Mexico 88003, USA.

${ }^{4}$ Institute for Applied Biosciences, New Mexico State University, Las Cruces, New Mexico 88003, USA. Correspondence and requests for materials should be addressed to I.A.H. (email: immoh@nmsu.edu).
} 
A principal feature in metazoan evolution is the loss of pathways for the biosynthesis of a group of nine essential amino acids (AAs) ${ }^{1}$. Concurrently, metazoans acquired effective mechanisms for the uptake of these and other AAs from their food, their distribution within the body, and the monitoring of intracellular and extracellular AA concentrations ${ }^{2,3}$.

A blood meal (BM) taken from a vertebrate host is the source of essential AAs for egg development in anautogenous mosquitoes $^{4}$. Post blood meal (PBM) females mosquitoes undergo complex changes in tissue-specific gene expression in order to transfer nutrient AAs from the digested meal to the developing oocytes, a process called vitellogenesis ${ }^{5,6}$. Vitellogenesis is triggered upon the initial detection of a surge of free AAs in the mosquito circulation ${ }^{7,8}$ and involves coordinated hormonal and nutritional regulation of enzymatic and transport processes in midgut $(\mathrm{GT})$, fat body $(\mathrm{FB})$ and ovaries $(\mathrm{OVs})^{8-10}$. The three subsequent key mechanisms of this process are: $\mathrm{BM}$ digestion in the GT, synthesis and secretion of yolk protein precursors by the $\mathrm{FB}$, and receptor-mediated uptake and deposition of yolk protein precursors and other nutrient reserves in developing oocytes.

Up to $19 \%$ of the total ingested AAs are used for the synthesis of soluble yolk proteins while the rest is largely used for energy production ${ }^{11-13}$. The digested AAs are transported across the apical and the basolateral membranes of the gut epithelium into the haemolymph and subsequently across the plasma membrane of FB cells. The yolk proteins synthesized by the FB are secreted into the haemolymph and are absorbed by the developing oocytes via receptor-mediated endocytosis ${ }^{14,15}$. Evidently, the membrane transport of free AAs is also necessary to supply a surge of protein synthesis in the OVs $\mathrm{PBM}^{16}$. Hence, the rapid redistribution of nutrient AAs during mosquito vitellogenesis requires highly regulated and efficient transport mechanisms. However, the molecular properties and regulation of such mechanisms are only partly explored.

To date, only a few transporters that mediate uptake of nutrient AAs in mosquitoes have been characterized ${ }^{2,17,18}$. Most of these are expressed in the larval stage and their role and significance in adult metabolism remains enigmatic. The aquatic larval stage of mosquitoes substantially differs from the terrestrial reproductive stage in terms of nutrient availability and metabolic demand $^{2,19}$.

In previous work, we have identified and characterized several AA transporters from the Solute Carrier Family 7 (SLC7) of Ae. aegypti, the yellow-fever mosquito. The SLC7 family includes two subgroups: the cationic AA transporters (CATs) and the heterodimeric AA transporters (HATs) ${ }^{20}$. RNA interferencemediated knockdown of 6 of the 11 members of the SLC7 family reduced target of rapamycin (TOR)-mediated nutrient signalling in the mosquito $\mathrm{FB}$, which resulted in limited egg production ${ }^{21,22}$. We cloned and characterized a first mosquito CAT, AaCAT1, and found that it is a $\mathrm{Na}^{+}$-independent transporter with a unique selectivity to L-histidine ${ }^{23}$. The AaCAT1 paralogue, AaSlif (=CAT3; GenBank accession \#: XP_001662274) is closely related to fruit fly slimfast (slif). Characterization of slif homologues in mosquito is of high interest as it may function as a mechanism combining transporter and receptor properties (transceptor). Drosophila slif is involved in signalling AA-availability in the metabolic regulation of fly growth ${ }^{24}$ and behavioural control of feeding arousal ${ }^{25}$. Both these functions comprise appealing targets for vector control. However, transport function of slif was unknown and could not be extrapolated from mammalian paralogues and insect CATs because of significant functional divergence of these transporters.

Here we describe the tissue-specific expression along with the biochemical-biophysical properties of AaSlif (=AaCAT3). Unexpectedly, our study revealed an unusual set of biophysical properties of this transporter including: dual-affinity for cationic and neutral AAs, lack of L-isomer selectivity, and a significant $\mathrm{Na}^{+}$-dependent component necessary for its high-affinity transport mode. These findings expand our current knowledge of transport functions and biological significance of the CAT-SLC7 mechanisms. The present studies also describe properties of a new AA transceptor with critical roles in nutrition and reproduction of an important disease vector.

\section{Results}

Cloning of $A a$ Slif and genomic structure of a CAT cluster. To determine the AaSlif cDNA sequence, we used Rapid Amplification of cDNA Ends ( $5^{\prime}$ RACE). We found an additional intron and an exon at the $5^{\prime}$-end of the transcript that was absent in the current annotation of the Ae. aegypti genome (VectorBase AAEL012131; see Supplementary Fig. 1). The AaSlif gene consists of five exons and four introns, which are spliced to an 1,881nucleotide-long open reading frame (ORF) encoding a 626-AA protein. When compared with the published genome sequence ${ }^{26}$ the AaSlif ORF contains 21 synonymous SNPs, which likely represent differences between the Liverpool (Genome sequence in VectorBase) and the Rockefeller strains (this study) of Ae. aegypti. The gene is located on the reverse strand of the supercontig 1.658 (Supplementary Fig. 1A). Two other genes of the AaCAT-SLC7 subfamily ( $A a \mathrm{CAT} 1$ and $A a \mathrm{CAT} 2$ ) are localized in a proximal reverse cluster downstream from AaSlif. The fourth gene of the AaCAT-SLC7 subfamily AAEL012129 is found in the forward strand and encodes the complete ORF of AaCAT4 (Supplementary Fig. 1A). AaCAT2 (AAEL012133) was only partly predicted in the current annotation of Aedes genome (Supplementary Fig. 1, brown splicing pattern). Our in silico analysis revealed a splicing scheme that is also supported by the gene expression pattern found in studies of the mosquito transcriptome (Supplementary Fig. 1, red + brown splicing pattern and magenta bars, respectively; based on the data from VectorBase).

Selection and conservation of the SBM in the Slif mechanism. In a previous phylogenetic study, we found that D. melanogaster slif (DmSilf) forms a phylogenetic cluster with two putative orthologues from Ae. aegypti, AaCAT1 and AaSlif ${ }^{23}$. Pairwise identity between DmSlif and AaCAT1 is $56.9 \%$; between the DmSlif and AaSlif $58.6 \%$, with 337 and 367 identical sites, respectively. To identify structure-function conservation among members of this cluster, we aligned the AA sequences of DmSlif orthologues from selected insect representatives against sequences and structural motifs of the recently crystalized prokaryotic relatives of the SLC7 family: the Methanocaldococcus jannaschii ApcT $^{27}$ and Escherichia coli AdiC ${ }^{28}$ (Supplementary Fig. 1B). In spite of only moderate pairwise identity, the prokaryotic and eukaryotic transporters aligned well in a region between the first and tenth transmembrane domain (TMD). The prokaryotic TMDs 11 and 12 aligned well to insect TMDs 11-12 as well as 13-14 with similar homology patterns (Supplementary Fig. 1B). The possibility of an alternative alignment supports the idea that the two C-terminal TMDs were duplicated in an universal CAT ancestor $^{23}$.

Figure 1 shows the alignment pattern of a putative substratebinding motif (SBM) interpolated from the EcAdiC threedimensional (3D) structure. It reveals both highly conserved and variable sites. Importantly, it defines that the predicted SBM residues are identical among dipteran slif orthologues and also are strongly conserved in the flour beetle (Tribolium castaneum), the body louse (Pediculus humanus corporis) and the honey bee (Apis mellifera) orthologues. 


\section{Consensus}

\section{Sequence Logo}

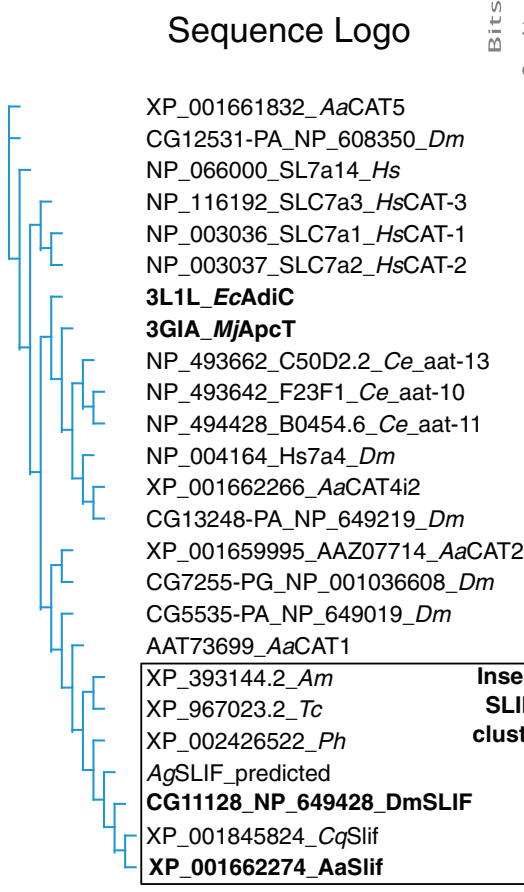

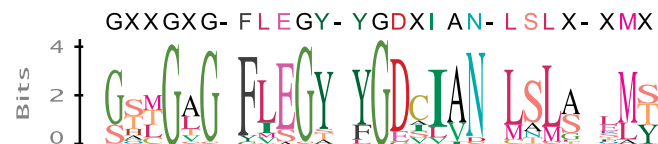

Transmembrane domains

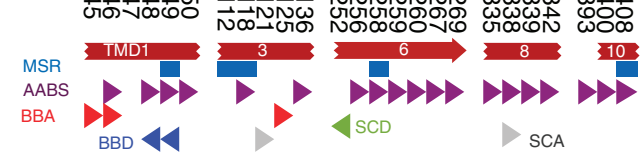

Figure 1 | Bioinformatics analysis of the AaSlif substrate-binding motif (SBM). Shown are a neighbour-joining consensus tree and an alignment of the putative SBM residues of selected CAT-SLC7 members. Putative Slif orthologues (outlined box) form a phylogenetic cluster with exactly one orthologue per selected insect genome and also show strong conservation of the SBM. The names of Drosophila and Aedes slif orthologues and their prokaryotic homologues used for the identification and alignment of SBMs are highlighted by bold font. Coloured and grey fonts depict variable and significantly conserved residues, respectively. The aligned numbers indicate the amino-acid positions in the AaSlif protein sequence. The partial SBM of the Tribolium Slif is due to its incomplete annotation. The coloured shapes depict specific putative components of the SBMs abbreviated as: AABS, amino acid-binding sites; BBA, backbone acceptor; BBD, backbone donor; MSR, mutation sensitive residues; SCA, side chain acceptor; SCD, side chain donor. Species abbreviations are: Aa, Aedes aegypti; Ce, Caenorhabditis elegans; Cq, Culex quinquefasciatus; Hs, Homo sapiens; Ph, Pediculus humanus; Tc, Tribolium castaneum.

Heterologous expression and electrophysiological characterization. The heterologous expression of the original AaSlif transcript (GenBank accession \#: KM593906) in Xenopus laevis oocyte resulted in a significant increase of Arg-induced currents compared with water-injected control oocytes $(I=10.2 \pm 5.8 \mathrm{nA}$ for $10 \mathrm{mM} \mathrm{L-Arg;} n>3, P=0.014 ; t$-test). However, such a signal was considered too weak for an electrophysiological characterization of the transport mechanism.

To improve AaSlif expression, we ordered synonymous optimized synthetic variants of AaSlif with a Xenopus-codon usage (synthesized by Genwiz Inc.) and cloned them in the pXOOM expression vector. Two plasmids: AaSlif co $_{\text {-pXOOM }}$ (GenBank accession \#: KM593906) and Aaslif co $_{\text {eGFP-pXOOM }}$ (GenBank accession \#: KM593907) both with the AaSlif ORF and the latter also fused to enhanced green fluorescent protein (eGFP) at the C-terminus, were tested.

The expression of AaSlif $\mathrm{co}_{\mathrm{co}} \mathrm{GFP}$ induced bright green fluorescent protein (GFP)-specific fluorescence that was correlated with a large inward current upon application of cationic AAs in oocytes on days $4-10$ after injection of cRNA $(\sim 10 \mathrm{nA}$ at $1 \mu \mathrm{M}$ and $\sim 100 \mathrm{nA}$ at $10 \mathrm{mM}$ of L-Arg; Fig. 2a). Importantly, the expression of $A a S l i f f_{c o}$ and $A a S l i f f_{c o}$ GFP resulted in no detectable differences in measured amplitude, saturable kinetics and voltage dependency; therefore, both variants could be used interchangeably in the analysis (Supplementary Fig. 2).
AaSlif has an apparent preference for Arg (Fig. 2b; Arg $>$ His $\approx$ Lys) without significant L-D enantiomer selectivity (Fig. 2c, current trace insert). AaSlif also generated significant inward currents on applications of L-citrulline (Cit) and L-ornithine (Orn), two metabolic AAs that we tested as intermediates of the nitric oxide (NO) synthesis and L-arginine recycling cascades (Supplementary Fig. 2). In contrast, betaine, $\gamma$-aminobutyric acid and taurine induced no significant responses. Notably, AaSlif also generates significant inward currents for neutral aliphatic (Ile, Leu, Met), aromatic (Phe, Trp, Tyr) and even acidic AAs (Supplementary Fig. 2).

Substrate saturation kinetics, $\mathrm{pH}$ dependency and uptake. A substrate saturation assay revealed that AaSlif has two apparent saturation points: one at $\sim 1 \mathrm{mM}$ and second at $\sim 20 \mathrm{mM}$ of L-Arg (Fig. 3a and Supplementary Fig. 3). The estimated $K_{\mathrm{d}}^{\mathrm{Hi}}$ and $K_{\mathrm{d}}^{\mathrm{Lo}}$ were $5.2 \pm 1.4 \mu \mathrm{M}$ and $6.9 \pm 0.8 \mathrm{mM}$ for $\mathrm{L}-\mathrm{Arg}$, $13.8 \pm 6.2 \mu \mathrm{M}$ and $6.8 \pm 2.6 \mathrm{mM}$ for $\mathrm{L}-\mathrm{Orn}$ and $15.6 \pm 8.5 \mu \mathrm{M}$ and $9.8 \pm 6.5 \mathrm{mM}$ for L-Phe. Our null hypothesis of a one point saturation model was rejected with $P<0.001, P=0.018$ and $P=0.034$ for $\mathrm{L}$-Arg, $\mathrm{L}$-Phe and L-Orn, respectively ( $t$-test). The amplitude of cationic and neutral AA-induced currents depended on the extracellular concentration of $\mathrm{Na}^{+}$, with apparent $\mathrm{Na}^{+}$ dissociation constant $K_{\mathrm{d}}=12.4 \pm 1.5$ and $13.8 \pm 2.2 \mathrm{mM}$ and Hill 
a

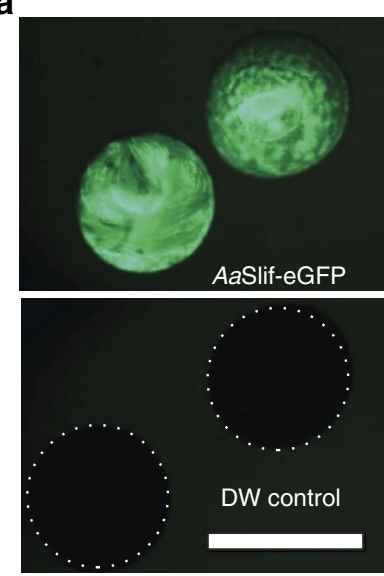

b
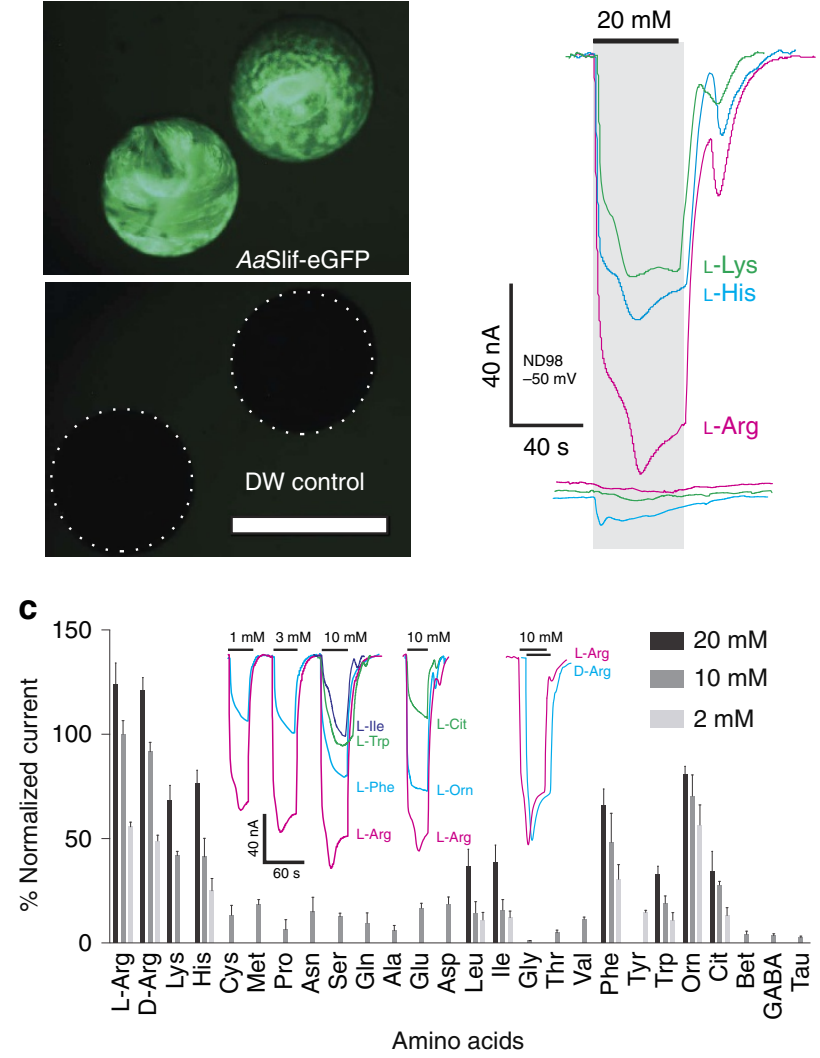

Figure 2 | Functional expression and AA-specificity profile of AaSlif.

(a) Functional expression of codon-optimized AaSlif-eGFP fusion protein showed bright fluorescence compared with control deionized waterinjected oocytes (bottom panel, white dashed outlines indicate position of control oocytes that are almost invisible with identical settings of the GFP-specific filter cube (Nikon 49583; EN GFP LP: Excitation Hq 470/40, Dichroic 495EM, Emission 500LP) and camera sensitivity); scale bar represents $1 \mathrm{~mm}$. (b) The expression of AaSlif correlates with large cationic AA-induced current responses. The coloured lines represent currents induced in the AaSlif-eGFP-expressing oocytes (top traces) versus control oocytes (bottom traces) after application of $20 \mathrm{mM}$ of L-enantiomer of Lysine $(K)$, Histidine $(H)$ and Arginine $(R)$. (c) AA specificity profile reconstructed from $A A$-induced currents. The bars are mean of percent normalized current + s.d. ( $n>2$ oocytes/samples per point) at 20, 10 and $2 \mathrm{mM}$ concentrations of AAs. Currents were recorded using two-electrode voltage clamp. The coloured traces show representative recordings of currents induced by three different concentration of L-Arg and L-Phe, as well as $10 \mathrm{mM}$ of L-Trp, L-lle, L-Arg, L-Orn and L-Cit. Oocytes were clamped to $-50 \mathrm{mV}$ holding voltage in a constant flow micro-chamber perfused at $\sim 2$-chamber volumes per second with ND98 or indicated solutions of AAs in $98 \mathrm{Na}^{+}$buffer. Currents were measured considering a steady-state current value and normalized to the mean of $10 \mathrm{mM} \mathrm{L-Arg} \mathrm{(=100 \% )}$ response in each oocyte for cross-oocyte comparison. Cit, citrulline; DW, deionized water; GABA, $\gamma$-aminobutyric acid; Orn, ornithine; Tau, taurine.

constant $\eta=1.8 \pm 0.6$ and $2.2 \pm 1.0$ at $1 \mathrm{mM}$ concentration of L-Arg and L-Phe, respectively (Fig. 3b). Acidification of the extracellular solution significantly increased the amplitudes of substrate-induced inward currents $(\sim 30-50 \%$ per $\mathrm{pH}$ unit; $P<0.001$ for $n>3$; $t$-test; Fig. $3 \mathrm{c}$ ). To define the transport activity of $A a S l i f$, we performed uptake assays with selected radioactive isotope-labelled substrates. The AaSlif expression increased the absorption of AAs in AaSlif-recombinant oocytes compared with deionized water-injected control oocytes. These results unequivocally verified that AaSlif functions as an AA transporter (Fig. 3d).
Cation dependency of AA-induced currents in AaSlif oocytes. Substitution of extracellular $\mathrm{Na}^{+}$by $\mathrm{K}^{+}$significantly reduced the Arg-induced current (Fig. 4a) and modified voltage dependency of AaSlif, shifting the inflection point towards neutral (Fig. 4b). Surprisingly, the $\mathrm{Na}^{+}-\mathrm{K}^{+}$substitution resulted in much lower current induced by Arg at concentrations below the $K_{\mathrm{d}}^{\mathrm{Lo}}$ (at 1 and $3 \mathrm{mM}$ of $\mathrm{L}-\mathrm{Arg}$, reduction $75 \pm 9.6 \%$ versus same in $\mathrm{Na}^{+}$media, $n>3$ ) in comparison to the current induced by same substrate above the $K_{\mathrm{d}}^{\mathrm{Lo}}$ (at $10 \mathrm{mM}$ of $\mathrm{L}-\mathrm{Arg}$, reduction $22 \pm 4.6 \%, n>3$ ). It suggests that at low AA concentrations Arg-coupled current has large $\mathrm{Na}^{+}$-coupled component, while it may become largely $\mathrm{Na}^{+}$independent at high concentrations of the organic substrate (Fig. 4a). The subtracted $I / V$ plots showed additional details regarding interaction of the AaSlif mechanism with $\mathrm{Na}^{+}{ }_{\mathrm{L}-\mathrm{Arg}}{ }^{+}$ ions (Fig. 4c). The data suggest that $\mathrm{Na}^{+}$ions have a much greater contribution in the substrate-induced current compared with $\mathrm{Arg}^{+}$. This effect is especially profound at transmembrane voltages below $-60 \mathrm{mV}$.

We also noticed that $\mathrm{Na}^{+}-\mathrm{K}^{+}$substitution delayed the recovery of the transporter after washing with $10 \mathrm{mM} \mathrm{L}$-Arg (Fig. 4a black arrow). The reduced recovery can be rescued by extracellular administration of 3 or $1 \mathrm{mM}$ L-Arg (Fig. 4a grey arrows). This indicates that AaSlif can be locked in some ion conductive state upon interaction with high concentration of L-Arg at low $\mathrm{Na}^{+}$or high $\mathrm{K}^{+}$levels. However, a more detailed analysis of this phenomenon was beyond the scope of the characterization.

Phenylalanine induced currents in AaSlif-expressing oocytes. The L-Phe was tested as a representative of neutral aromatic substrates. Its application resulted in a substrate-induced inward current (Figs $2 \mathrm{c}$ and $3 \mathrm{a}, \mathrm{b})$. The Phe-induced current was strictly $\mathrm{Na}^{+}$-dependent. $\mathrm{Na}^{+}$ions cannot be substituted by $\mathrm{NMDG}^{+}$ ( $N$-methyl D-gluconate) or $\mathrm{Li}^{+}$(Fig. 4d-f). Interestingly, the substitution of $\mathrm{Na}^{+}$with $\mathrm{K}^{+}$resulted in a reversion of the Phe-induced current, which implies the presence of some cationic efflux or blockage of cationic influx, for example, leak current (Fig. $4 \mathrm{~d}, \mathrm{e}) . \mathrm{I} / \mathrm{V}$ plots for $\mathrm{L}-\mathrm{Phe}$ with $\mathrm{K}^{+}$display a characteristic nonlinearity with an inflection point close to $-70 \mathrm{mV}$ (Fig. $4 \mathrm{e}$ ).

AaSlif expression and regulation PBM. To reveal spatiotemporal expression and regulation of AaSlif, we measured the accumulation/retention of its transcript in whole body, selected body parts and organ samples isolated from non-blood fed (control) and blood fed mosquito females. The samples were isolated and tested after 3, 12, 24, 48, 72 and 96-h PBM intervals. The AaSlif gene showed a varied and rapidly regulated expression profile (Fig. 5 and Supplementary Fig. 4). Specifically, it was significantly upregulated in whole females at 3,12 and $72 \mathrm{~h} \mathrm{PBM}(\sim 10,10$, 60 times, respectively; $P<0.001$; two-way analysis of variance followed by Tukey's honest significant difference (HSD) post-hoc tests for $n=3$ samples). However, AaSlif expression declines in the whole body samples 24 and $48 \mathrm{~h}$ PBM close to the expression levels in the corresponding control samples. The elevated expression in $12 \mathrm{~h}$ PBM samples correlates with the intensified transcription of AaSlif in the FBs and thorax, as well as moderately elevated transcription in the OVs and Malpighian tubules (MTs). In contrast, $72 \mathrm{~h}$ PBM samples showed strong overexpression across all selected body part and organs, including the mosquito gut. The level of transcript decreased in the 96-h PBM samples, except for OVs (Fig. 5).

\section{Discussion}

The requirement for acquisition of essential AAs through blood feeding makes anautogenous Ae. aegypti mosquitoes an effective 
a

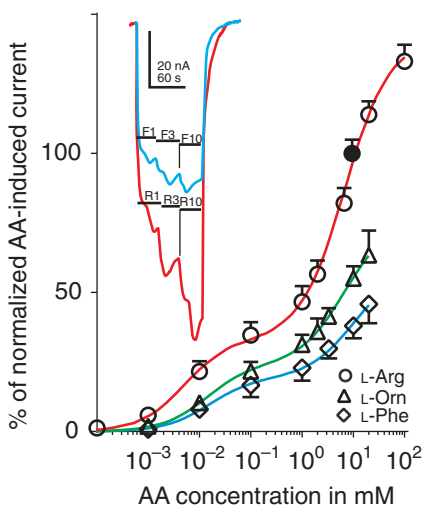

b

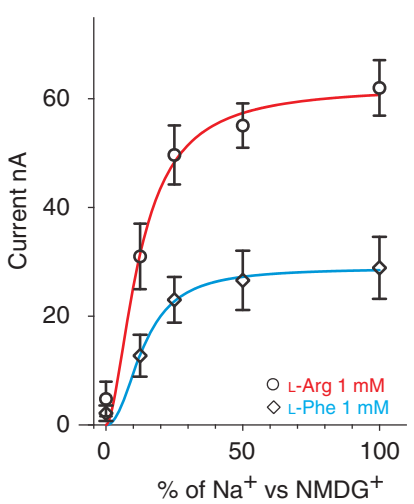

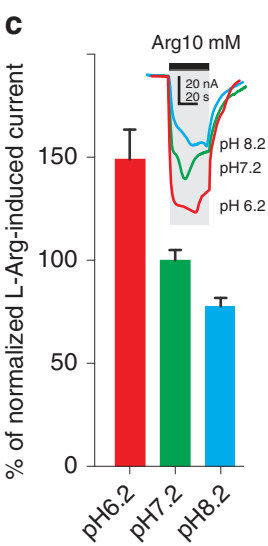

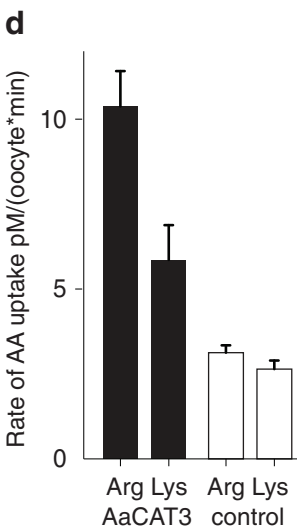

Figure 3 | AaSlif substrate saturation kinetics, pH dependency and substrate specificity. (a) Saturation kinetic graphs for L-Arg-, L-Phe- and L-Orn-induced currents. The insert shows the relative amplitudes of L-Arg and L-Phe-induced currents in AaSlif-expressing oocytes stimulated with three different concentrations of L-Arg and L-Phe. The data were normalized between different oocytes using mean value responses to $10 \mathrm{mM}$ L-Arg (Filled circle point). Data are mean \pm s.d. for $n>3$. (b) Sodium saturation kinetics for $1 \mathrm{mM}$ L-Arg- and L-Phe-induced currents. Data are mean \pm s.d. for $n=3$. (c) pH dependency of $10 \mathrm{mM}$ L-Arg-induced currents. Bars are mean + s.d. for $n=3$ oocyte/samples, that are significantly different $P>0.001 ; t$-test. Insert shows a scaled superposition of typical L-Arg-induced currents induced upon sequential application of L-Arg at specified pH values. (d) Uptake of radiolabelled cationic AAs in AaSlif-expressing oocytes (black) versus control oocytes (white). Data are mean + s.d. for $n=5$ individual oocyte assays.

vector of important arboviral diseases, including dengue, yellow fever and Chikungunya ${ }^{29}$. BM-derived AAs are a major source of energy and building blocks during mosquito reproduction. Consequently, mosquitoes possess a highly efficient and tightly regulated system to distribute AAs between different tissues ${ }^{21}$. This transport system is only partly understood. In this study, we functionally expressed and characterized a mosquito orthologue of Drosophila slif, AaSlif. This transporter represents the first characterized member of the unique insect-specific cluster of the CAT-SLC7 subfamily of AA transporters ${ }^{23,30}$ and the first characterized representative of a new transport system, SLIF.

The CAT acronym was originally coined for a group of mammalian AA transporters of the canonical $y^{+}$system, a Na ${ }^{+}$. independent transport mechanism selective for cationic $\mathrm{L}-\mathrm{AAs}^{20,31}$. The first CAT cloned and characterized via recombinant expression in Xenopus oocytes was serendipitously identified as a murine leukemia virus receptor with an explicit homology to yeast AA permeases and properties of the canonical $\mathrm{y}^{+}$(CAT) system ${ }^{27,28}$. Subsequent characterization of three from four existing mammalian members of the CAT-SLC7 subfamily showed similar AA selectivity patterns with some variations in affinity and tissue expression ${ }^{32}$. Our previous phylogenomic analysis of the CAT-SLC7 family showed that mammalian and putative insect CATs share a common ancestral root $^{23}$. However, insect transporters formed independent clusters suggesting specificity of adaptations of CAT mechanisms in mammals and insects ${ }^{23}$. An important finding from the extended bioinformatics analysis of the present study is that there is only one slif orthologue per insect genome. Also, the putative SBMs of slif orthologues in fruit flies and mosquitoes are identical even though these two insect groups diverged around $0.26 \mathrm{Bya}^{33}$ (Fig. 1). These evidences strongly suggest that the SBM, transport mechanism and biological functions of these transporters have undergone strong stabilizing ( = purifying) selection. Another important finding is that SBMs of slif orthologues are distinct from the SBM of the previously characterized AaCAT1 (ref. 23) and other mammalian and insect CATs (Fig. 1). These facts are consistent with the hypothesis that the slif mechanism plays a unique role in insect metabolism and lacks genetic and functional redundancy.

Previously, three mosquito CATs, AaCAT1, AaCAT2 and AaSlif (=AaCAT3), were identified as components of the $\mathrm{FB}$ nutrient sensor system that uses the TOR signalling pathway to activate reproductive processes in mosquito females after blood consumption ${ }^{21,22}$. In the present study, we show that this trio plus AaCAT4 is physically co-localized in the mosquito genome (Supplementary Fig. 1). We also found comparable transcripts of these CATs in the transcriptomic libraries at VectorBase ${ }^{34}$ (Supplementary Fig. 1A, magenta pattern), supporting the notion that all four are functional genes. The tight clustering pattern suggest that CATs may be under a unified genetic control. However, considering the diverse SBMs we found in these transporters, the anticipated contributions in AA transport and signalling are clearly different. The genomic organization, together with the phylogenetic data (Fig. 1) and functional data (this study and ref. 22), suggests that the CAT cluster in the Ae. aegypti genome is the result of gene duplications and subsequent functional adaptation of individual CATs for a specific physiological role, which may include a substrate specialization as found in AaCAT1 and AaSlif.

Using codon-optimized transcripts, we achieved a significantly better expression of AaSlif in Xenopus oocytes compared with wild-type transcript providing a unique opportunity for high-resolution electrophysiological characterization of this transporter. Chimeric fusion of AaSlif with eGFP did not modify expression and functional properties of this transporter (Fig. 2a) and could be used for the visual monitoring of CAT proteins in heterologous or naïve expression systems without functional artifacts.

Our functional characterization reveals that AaSlif is a high-efficiency carrier of cationic AAs with particular preference to Arg (Figs 2 and 3). It also transports urea cycle metabolites that represent products of the NO synthesis pathway ${ }^{35}$. These characteristics of AaSlif are consistent with the generic properties of mammalian CAT-SLC7 transporters ${ }^{36}$. Nonetheless, the AaSlif mechanism differs from the $\mathrm{y}^{+}$system of previously characterized mammalian CATs ${ }^{32}$ and the His-specific mosquito AaCAT1 (ref. 23). AaSlif showed a substantially broader substrate spectrum compared with previously characterized CATs. Moreover, it equally transports $\mathrm{L}$ - and D-enantiomers of Arg (Fig. 2c); whereas the mammalian CATs are strictly or significantly stereo-selective ${ }^{37,38}$. It also generates significant inward currents for neutral aliphatic (Ile, Leu, Met), aromatic (Phe, Trp, Tyr) and even acidic AAs (Fig. 2c). Such currents cannot be explained by facilitated diffusion of neutral AAs or 
a
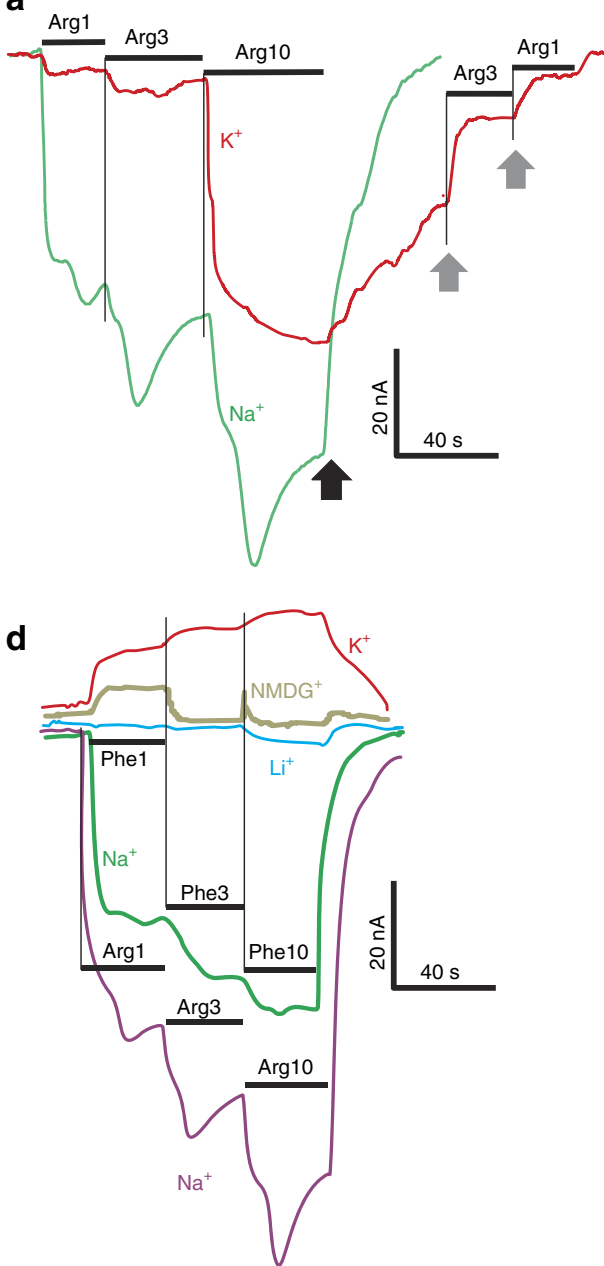

b

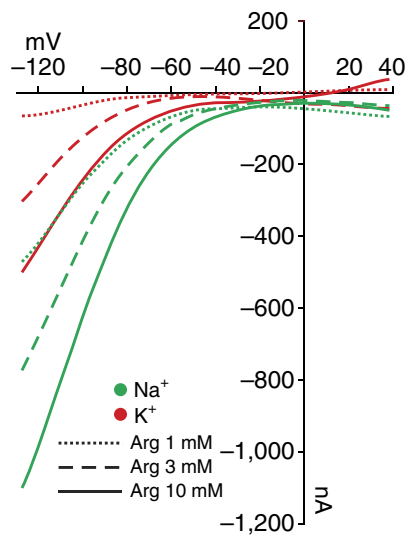

e

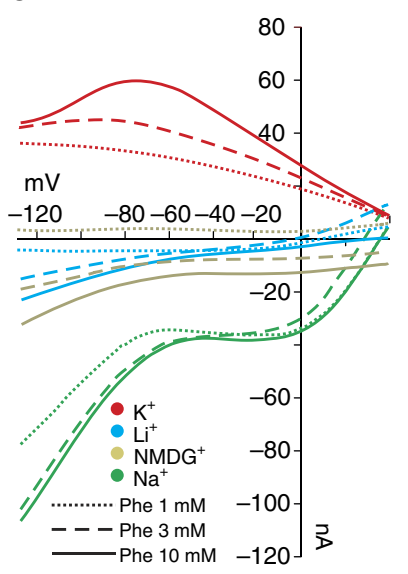

C

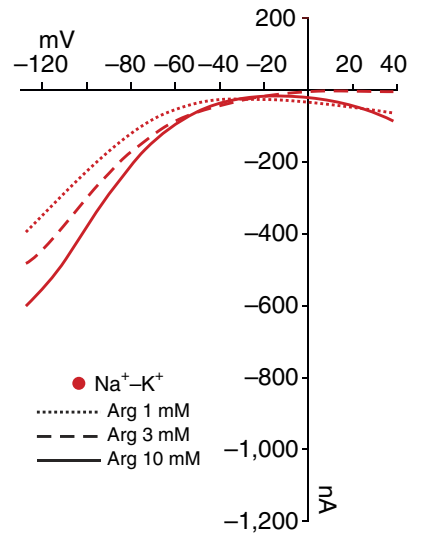

f

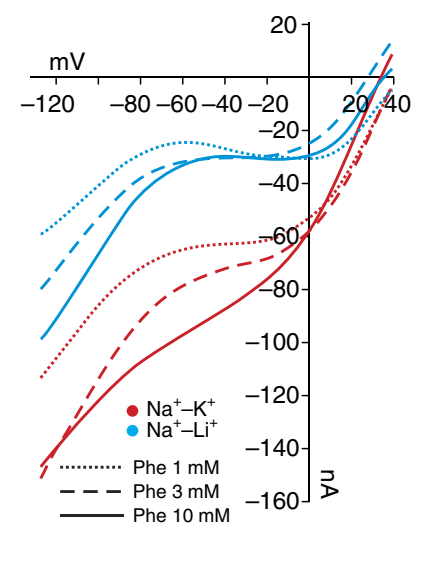

Figure 4 | Cation dependency of AA-induced currents. (a) Example of substrate-induced currents in an AaSlif-expressing oocyte after application of 1, 3 and $10 \mathrm{mM}$ of $\mathrm{L}-\mathrm{Arg}$ in $98 \mathrm{Na}^{+}$(green line) and $98 \mathrm{~K}^{+}$(red line) buffers (containing identical concentrations of $\mathrm{Cl}^{-}$and other ions). Black arrow indicates washing after L-Arg application with initial buffer saline. Grey arrows indicate accelerated recovery after re-application of 3 or $1 \mathrm{mM}$ of L-Arg (for $98 \mathrm{~K}+$ buffer only). (b) Background-subtracted I/V plots of AaSlif-expressing oocytes in $98 \mathrm{Na}^{+}$and $98 \mathrm{~K}^{+}$buffers with three different concentrations of L-Arg (insert). (c) I/V plot calculated by subtraction of the current values measured in $98 \mathrm{~K}^{+}$from those in $98 \mathrm{Na}^{+}$. Results show no significant dependency of current on L-Arg concentration. (d) L-Phe-induced currents in four different buffers. Results show that substitution of $\mathrm{Na}^{+}$with $\mathrm{Li}^{+}$and $\mathrm{NMDG}^{+}$ions reduced substrate-induced currents, whereas the substitution with $\mathrm{K}^{+}$reversed the current. (e) I/V plots acquired from the same oocyte as in $\mathbf{d}$ with different cation compositions, as colour-coded in insert. (f) $\mathrm{I} / \mathrm{V}$ plots calculated by subtraction of current values measured in $98 \mathrm{~K}^{+}$and $98 \mathrm{Li}^{+}$buffers from those in $98 \mathrm{Na}+$ buffer.

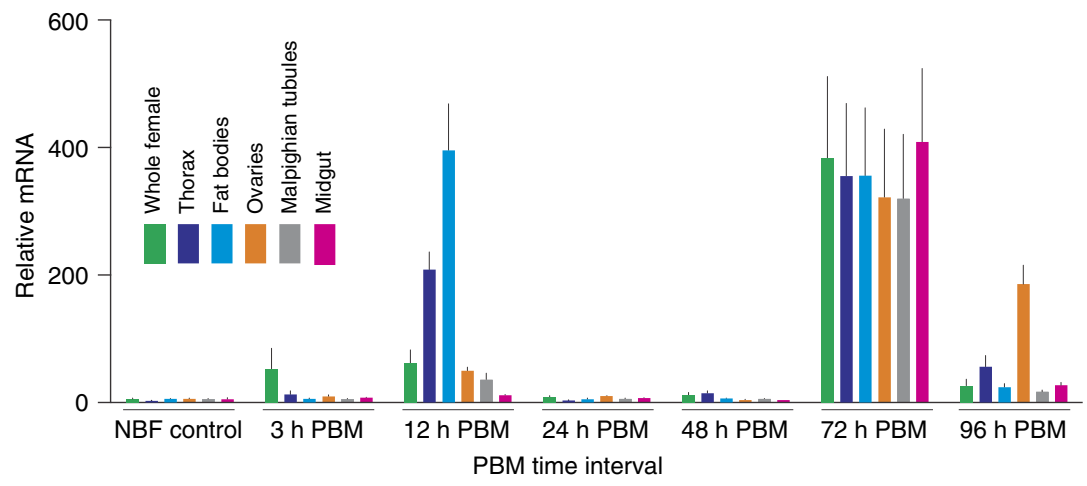

Figure 5 | AaSlif expression and regulation in selected mosquito tissues and organs. The transcript levels were determined using quantitative PCR (qPCR). The data represent relative quantities of AaSlif transcript that were normalized with qPCR levels of ribosomal protein S7 (rpS7) mRNA in the same tissue sample set. Bars are means + s.e.m. for $n=3$ replicates collected from different sets of mosquitoes (analysis of variance followed by Tukey's HSD post hoc test). The RNA samples were isolated from whole body and various body parts and organs of adult female mosquitoes grouped as shown by the colour-coding insert. The horizontal scale indicates group-specific conditions: non-blood fed (NBF, control), 3, 12, 24, 48, 72 and $96 \mathrm{~h}$ post blood meal (PBM). 
cationic/neutral AA coupled exchange mechanisms found in mammalian CAT and HAT members of the SLC7 family ${ }^{23,30}$. Such mechanisms should generate no outward currents in responses to neutral AAs. The ion substitution assay showed that the inward neutral AA-induced current is due to involvement of inorganic ions (Fig. 4d-f).

Unexpectedly, we observed complex saturation kinetics of the AaSlif consistent with an unusual dual-affinity mechanism and two dissociation constants: one in low-micromolar range and other in low-millimolar ranges (Fig. 3). Notably, its substrateinduced currents were $\mathrm{pH}$-dependent and correlate with the uptake of radiolabelled substrates (Fig. 3c,d, respectively). Moreover, the high-affinity mode of the AaSlif interaction with L-Arg was largely $\mathrm{Na}^{+}$dependent (Fig. $4 \mathrm{a}-\mathrm{c}$ ). The $\mathrm{Na}^{+}$-coupling was also associated with the neutral AA-induced inward current. This revealed a Na ${ }^{+}$selectivity of the AaSlif mechanism that neither $\mathrm{Li}^{+}$nor NMDG ${ }^{+}$ions can substitute (Fig. 4). Interestingly, substitution of $\mathrm{Na}^{+}$with $\mathrm{K}^{+}$resulted in an inversion of the neutral AA-induced currents that was not observed upon $\mathrm{Na}^{+}$ substitution with $\mathrm{Li}^{+}$or NMDG ${ }^{+}$(Fig. $4 \mathrm{~d}-\mathrm{f}$ ). This indicates that $\mathrm{K}^{+}$is a potent modulator of ion fluxes through AaSlif, a finding that requires further experimental analysis.

We propose that the identified properties represent adaptations of AaSlif for its action in an extended range of AA concentrations. At low AA availability, for example, during the state-of-arrest before the mosquito takes a BM, it acts as secondary high-affinity transporter using $\mathrm{Na}^{+}$motive forces for the intracellular accumulation of AAs. In contrast, at high AA availability, for example, after a BM, it acts as a low affinity, passive uniporter facilitating transmembrane diffusion of nutrient substrates without using the electrochemical energy of ion gradients. Dual-affinity mechanisms have never previously been reported among metazoan representatives of the Major Facilitator Superfamily. However, a dual-affinity nitrate transporter (CHL1, also NRT1) has been recently reported among MFS representatives in plants ${ }^{39}$. When phosphorylated at Thr101, CHL1 acts as a high-affinity nitrate transporter, whereas dephosphorylated transporter has low affinity. It has been suggested that switching involves dimerization, and that dual affinity CHL1 represents adaptation for managing highly variable concentrations of critical nutrients ${ }^{40}$.

AaSlif utilizes $\mathrm{Na}^{+}$motive forces for the transport of neural AAs and also the high-affinity transport of cationic AAs. Such a coupling satisfies thermodynamic requirements for intracellular translocation of substrates from low-concentration domains. The identification of the first CAT with explicit sodium dependency is truly remarkable for the field of transport biology. Although mammalian CATs generally do not use inorganic cations for translocation of cationic AAs, some $\mathrm{Na}^{+}$dependency has been reported for representatives of the $\mathrm{y}^{+}$system. For example, the first characterized murine CAT transports Cys and homoserine only in the presence of $\mathrm{Na}^{+}$(ref. 41). Heterologous expressed human CAT-3 has been shown to mediate $\mathrm{Na}^{+}$as well as $\mathrm{K}^{+}$ conductance ${ }^{42} \cdot \mathrm{Na}^{+}$dependency has also been reported in some HAT-SLC7 transporters resembling the $\mathrm{y}^{+} \mathrm{L}$ transport system ${ }^{32}$.

In this work, we have achieved unprecedented resolution for electrophysiological characterization of a CAT mechanism and tested a more comprehensive set of substrates compared with previous reports. Hence, it is possible that previous electrophysiological characterization of CAT mechanisms were simply incomplete. Alternatively, it is possible that a majority of vertebrate CATs substantially deviate from the insect slif type mechanism in such a way that these transporters either were never $\mathrm{Na}^{+}$coupled or secondarily lost the $\mathrm{Na}^{+}$coupling. Such a possibility received indirect support from the comparative analysis of SBMs, which showed additional negatively charged glutamic acid residues in the AA position 121 (Fig. 1). Such negatively charged SBMs are typical for $\mathrm{Na}^{+}$-coupled transport mechanisms. Additional analysis of the AaSlif mechanism could gain important insights about the molecular evolution and structural adaptations of CATs.

AAs play an important role as signalling molecules in mosquito vitellogenesis. Rising levels after a BM activate the TOR signalling pathway in GT, FB and OVs promoting tissue-specific reactions ${ }^{8,10,43-46}$. TOR signalling in other key tissues involved in vitellogenesis like nervous system and MTs has not yet been examined but it seems likely that these tissues are able to receive and process AA signals, too.

The AaSlif gene showed tissue-specific expression that was upregulated by BM with 12 and $72 \mathrm{~h}$ spikes in several of the tissues we examined (Fig. 5). The upregulation of AaSlif at $12 \mathrm{~h}$ precedes the peak of digestive enzyme activities in the GT and the peak in AA concentration in the Ae. aegypti haemolymph that coincide between 24 and $30 \mathrm{~h} \mathrm{PBM}{ }^{19}$. This suggests that female mosquitoes turn up the AaSlif mechanism to achieve rapid directed delivery of AAs to FB and OVs in times of abundance (PBM) and also to balance sufficient intracellular levels of AAs during limited accessibility of these substrates when haemolymph AA levels decline. The rather modest upregulation of AaSlif in the GT PBM is surprising and deserves additional studies. A possible explanation is that AaSlif may act through its $\mathrm{Na}^{+}$-independent high-throughput mode at this time in this tissue. Alternatively, the GT may utilize another group of AA transport systems than FB or OVs to shuttle the large amounts of AAs into the haemolymph during this particular time point.

The high AaSlif expression in all tissues we found at $72 \mathrm{~h} \mathrm{PBM}$ is surprising because at this time the digestion of the $\mathrm{BM}$ is already at its end and AA transport between tissues is thought to be declining. We have earlier shown similar transcription upregulation during late vitellogenesis for several other SLC7type AA transporters, specifically from the HAT family ${ }^{21}$. The gradual reduction of free AA levels at this time might trigger the overexpression of these transporters. In the case of AaSlif, this could compensate for a substrate concentration-dependent switch from its fast low-affinity transport mode to the slow high-affinity mode. An alternative explanation is that these proteins are synthesized in order to allow a quick response to the rapid changes in AA concentrations associated with a second BM. To fully understand the biological relevance of this late upregulation phenomenon it would be interesting to determine the changes in the metabolomes and transportomes in these tissues over the cause of vitellogenesis with a comprehensive transcriptomics/ metabolomics survey.

The AaSlif properties and expression profile also support its contribution to AAs signalling in the alimentary and reproductive tissues. Such a contribution is evident from our previous evaluation of the role of CATs in mosquito reproduction ${ }^{21,22}$. This is also supported indirectly by data from studies of the slif mechanism in Drosophila. FB-specific suppression of DmSlif resulted in a global growth defect, similar to that seen in larvae grown under low-nutrient conditions ${ }^{24,47}$. Slif knockdown in the dopaminergic neurons of the Drosophila brain resulted in a strong inhibition of feeding 25 . This phenotype was associated with the General Control Nonderepressing 2-mediated neuronal detection and behavioural rejection of AA-imbalanced meals by fly larvae. Therefore, slif orthologues play an important role in the modulation of insect appetite and dietary source preferences.

An interesting question that warrants further research is to what degree AaSlif is involved in the NO signalling pathway in the mosquito $\mathrm{FB}$. NO is an important activator of the insect innate immune system and involved in the control of bacterial and protozoan parasites ${ }^{48,49}$. Nitric oxide synthase catalyzes the 
synthesis of $\mathrm{NO}$ and the byproduct L-citrullin from its substrate $\mathrm{L}-\mathrm{Arg}^{50}$. Both, L-citrullin and L-Arg are also substrates of AaSlif. Therefore, it is possible if not likely that AaSlif plays a part in the control of NO synthesis by regulating intracellular L-Arg levels and thereby controlling NO synthase activity with consequences for the insect immune system and mosquito vectorial capacity.

$A a S l i f$ is the first characterized metazoan transporter with dual affinity for cationic and neutral AAs with a $\mathrm{Na}^{+}$-dependent component. Based on its unique properties and strong evolutionary conservation, we propose to define insect-specific orthologues of AaSlif as a new AA transport system, SLIF.

Expression profiles and transport kinetics suggest that AaSlif functions as a high-efficiency transporter genetically and biophysically adapted to act in diverse habitats where unautogenous mosquitoes experience extremely variable availability of nutrient AAs, ranging from 'very limited' during a BM-preceding period to 'over-saturated' after a BM.

Arthropod-vectored infectious diseases put billions of people at risk worldwide. Novel means to control vector and pests populations are urgently needed. Our study reveals a new insect-specific transceptor for essential AAs, thereby opening new venues for the development of selective and environmentally safe control methods targeting appetite, development and reproduction in pest and vector insects.

\section{Methods}

Experimental animals. Ae. aegypti (Rockefeller strain) was used in all experiments. The mosquito population was maintained in the insectary of the Molecular Disease Vector Physiology Laboratory at New Mexico State University. Mosquitoes with high nutrient reserves were used for all experiments ${ }^{51}$. Larvae were reared using 'Special Kitty' cat food (Walmart). Adults were housed in cube-shaped cage ( $30 \mathrm{~cm}$ each side) with $20 \%$ sucrose solution at $26.5^{\circ} \mathrm{C}, 70 \% \mathrm{RH}$ and 16:8 h (light/dark) cycle. Chickens (Gallus gallus domesticus) were used to feed adult female mosquitoes, following the guidelines of the Institutional Animal Care and Use Committee (IACUC) of the New Mexico State University under approved protocol (\#2011-041).

Bioinformatic analysis. For combined phylogenomic-structural analysis, the NCBI protein databases were screened for slif homologues using BLASTp. A set of protein sequences that were considered important and sufficient for comparative analysis of structural and evolutionary aspects of SLC7-CAT mechanisms was selected. Insect genomes were selected based on their taxonomic reciprocity, as well as quality and completes of an existed annotations. The protein sequences undergone multiple sequence alignment with MEGA $^{52}$ implementation of the MUSCLE algorithm ${ }^{53}$ followed by manual correction. The transmembrane helices, known mutation-sensitive site and substrate interaction sites were interpolated from a consensus alignment of selected CATs with crystallized prokaryotic relative from Methanocaldococcus jannaschii, $\mathrm{MjApcT}^{27}$ and Escherichia coli, EcAdiC ${ }^{28}$ (Supplementary Fig. 1B). The TMDs 11-14 were defined by using a TMD prediction algorithm and manual improvement. The coordinate file of bacterial proteins structure (3GIA, 3OB6 and 3L1L) was also used to build 3D models of AeCAT3 in L-arginine occlusion and outside open conformation that was used for docking of L-arginine and identification of additional substrate interaction residues integrated in the final Figures. The Yasara ${ }^{54}$ structure software and MOE LigX ${ }^{55}$ algorithm were used for reconstruction of the $3 \mathrm{D}$ model and substrate interaction pattern, respectively. The identified residues position were trimmed from the complete alignment and used for preparing of SBMs diagram using Generous 6 software package ${ }^{56}$.

The evolutionary history was analysed using the neighbour-joining, minimum evolution, maximum parsimony and Bayesian methods that produced similar tree topologies. The neighbour-joining tree was selected as a more common representation of the evolutionary history of transport proteins. The tree was visualized using FigTree 1.3.1 software (A. Rambaut). It was drawn with estimated branch lengths used to infer the tree relative to a 3.7-billion-year-long scale from the root.

Molecular cloning and RACE verification. Total RNA was isolated from female mosquito $12 \mathrm{~h}$ after blood feeding using Trizol (Life Technologies). First-strand cDNA was synthesized on $1 \mu \mathrm{g}$ of total RNA using Omniscript RT kit (QIAGEN). The AaCAT3 ORF was PCR amplified based on the predicted ORF of AAEL012131RA gene (VectorBASE) using primers containing BamHI site for upstream primer or EcoRI site for downstream primer, cloned into pCR2.1 TOPO (Life Technologies) and sequenced. The Aaslif (AaCAT3) ORF was subcloned into an expression vector pXOOM. As we observed low expression signal with this construct, we tested for a possibility of alternative $5^{\prime}$ extension of Aaslif ORF in mosquito genome using $5^{\prime}$
RACE. cDNA was synthesized using SMARTer RACE Amplification Kit (Clonetech). One microgram of RNA isolated from $12 \mathrm{~h}$ PBM females was used for the synthesis with $5^{\prime}$-CDS primer A and followed the product manual.

Gene-specific RACE PCR and nested PCR primers were designed using the $5^{\prime}$ sequence of AAEL012131-RA (VectorBase, for sequences see Supplementary Table 1). RACE PCR was performed with a gene-specific primer and Universal Primer A Mix or Nested Universal Primer A. RACE PCR products were separated by agarose gel electrophoresis, and bands were excised for gel extraction by QIAGEN Gel Extraction kit (QIAGEN). Gel-extracted PCR products were cloned into pCR2.1 TOPO vector using TOPO TA Cloning Kit (Life Technologies). Resulted $5^{\prime}$ end sequence was used as a query for local BLASTn against Ae. aegypti genome sequence (AaegL2, VectorBase) to find the genomic locus, and exonintron boundary was manually adjusted so that the intron contains canonical splice site (GT-AG).

As we did not identify alternatively spliced variant, we concluded that the low expression was due to suboptimal translation in heterologous system and subsequently optimized AaSlif codon usage for expression in X. laevis oocyte. Specifically, the Xenopus-optimized AaSlif was synthesized using GENEWIZ service. We also made a C-terminus fusion of AaSlif with eGFP reporter for monitoring the levels of expression and appropriate trafficking of the AaSlif in individual oocytes. The AaSlif (with or without eGFP) sequence was PCR amplified and cloned into pCR2.1 TOPO (Life Technologies) and restriction-digested insert was subcloned into the expression vector pXOOM using restriction sites BamHI (on $5^{\prime}$ ) and NotI (on $3^{\prime}$ ).

Heterologous expression and characterization. cRNA for heterologous expression was synthesized by in vitro transcription of XbaI-linearized AaslifcopXOOM and AaSlifcoeGFP-pXOOM plasmids using the mMessage-mMachine (Ambion Inc.). The integrity and quantity of the RNA was confirmed by agarose gel electrophoresis. Surgically isolated, collagenase-treated and defolliculated stage V-VI X. laevis oocytes were purchased from Ecocyte Bioscience US LLC and injected with $\sim 35 \mathrm{ng}$ of AaSlif cRNA using Nanoliter 2000 injector (WPI). Oocytes were incubated for $2-6$ days at $17^{\circ} \mathrm{C}$ in sterile $98 \mathrm{mM} \mathrm{Na}^{+}$oocyte media supplemented with $2.5 \mathrm{mM}$ sodium pyruvate, $100 \mathrm{U} \mathrm{ml}^{-1}$ penicillin, $100 \mathrm{mg} \mathrm{ml}^{-1}$ streptomycin and $5 \%$ horse serum. For ion dependency assay, the $98 \mathrm{mM} \mathrm{Na}^{+}$was substituted with the equimolar amount of $\mathrm{K}^{+}, \mathrm{Li}^{+}$or $\mathrm{NMDG}^{+}$. The exact recipes for solution preparation are summarized in the Supplementary Table 1. The substrate-induced currents were recorded from voltage clamped oocytes at constant flow perfusion. The holding voltage was $-50 \mathrm{mV}$, except for ramp stimulation. The $I / V$ data sets were acquired during ramp stimulation (step $-130 \mathrm{mV}$; $200 \mathrm{~ms}$, ramp -130 to $+40 \mathrm{mV}$ in $1 \mathrm{~s}$; Supplementary Fig. $2 \mathrm{~A}$ ). The $I / V$ recording ( $1 \mathrm{kHz}$ sampling) was filtered with digital $50 \mathrm{~Hz}$ low pass 8 pole Bessel filter, reduced by factor 30 (substitute average) and used to build $I / V$ plots. The $I / V$ before substrate application was subtracted to eliminate substrateindependent current component. Additional corrections include subtraction of baseline drift current, which reflects slow adaptation of ion pumps after ion substitution (typically $<5 \mathrm{nA}$ ), if required. The data (mean \pm s.d., $n>2$ oocyte/ sample for each point) were fit with a preferable two-site specific binding model: Site $1=\mathrm{BmaxHi}^{\star} X /\left(K_{\mathrm{d}}^{\mathrm{Hi}}+X\right)$, Site $2=\mathrm{BmaxLo}^{\star} X /\left(K_{\mathrm{d}}^{\mathrm{Lo}}+X\right)$ and $Y=$ Site $1+$ Site 2 , where Bmax is apparent saturation current for high- and low-affinity components, respectively, $X$ are actual current values and $K_{\mathrm{d}}$ are apparent dissociation constants. One site-specific binding with Hill slope model was used as an Null hypothesis in evaluation of organic substrate-binding kinetic and for approximation of $\mathrm{Na}^{+}$ binding data: $Y=\operatorname{Bmax}^{\star} X \eta /\left(K_{\mathrm{d}} \eta+X \eta\right)$, where $\eta$ is apparent Hill constant.

Radiolabelled amino-acid uptake assay. AaSlif-expressing and control oocytes were conditioned in AA-free ND98 for 3-4 h and placed in $600 \mu \mathrm{l}$ of $98 \mathrm{Na}^{+}$media containing $2 \mathrm{mM}$ of final concentration of L-Arg and L-Lys with added $10 \mu \mathrm{l}$ of $0.1 \mu \mathrm{Ci} \mu \mathrm{l}^{-1}$ of L-[14C(U)]-Arg or $1 \mu \mathrm{Ci} \mu^{-1}$ of L-[4,5-3H(N)]-Lys, respectively. After incubation for $20 \mathrm{~min}$ at room temperature, oocytes were individually rinsed twice with excessive volume of ice-cold $98 \mathrm{Na}^{+}$media and lysed in $0.2 \mathrm{ml}$ of $1 \%$ SDS solution with ultrasonic treatment. The individual samples were diluted with $2 \mathrm{ml}$ of scintillation liquid and radioactivity was measured using a Beckman-Coulter LS6500 scintillation counter (Beckman). The AA uptake rate was extrapolated considering the isotope-labelled amounts, final concentrations and specific activities of AAs (SA L-Arg, 0.31 and L-Lys, $45 \mathrm{Ci} \mathrm{mmol}^{-1}$, Moravek Biochemicals). Disintegration per minute $(\mathrm{DPM})$ data were converted to $\mathrm{mmol} \mathrm{min}^{-1}$ using a constant $(1 \mathrm{Ci}=2.22$ $\times 1012 \mathrm{DPM}): \mathrm{mmol} \mathrm{min}^{-1}=(\mathrm{DPM} \times$ molar ratio [all/isotope] $) /(2.2 \times 1012 \times$ $\mathrm{SA} \times 20)$. Final data are shown in $\mathrm{pmol} \mathrm{min}^{-1}$.

Statistical analysis. Each experiment was repeated at least three times, using oocyte batches from different isolations. Statistical significance in the electrophysiology experiments was determined by unpaired Student's $t$-test. All underlying assumptions of the test were met by the data. For quantitative PCR, triplicate samples prepared from different mosquito individuals were analyzed. Two-way analysis of variance followed by Tukey's HSD post-hoc test was used to validate significant differences of a spatio-temporal gene expression. The data met the assumptions of the test. The PRIZM5 (GraphPad Software, Inc.) and SigmaPlot/SigmaStat (Systat Software, Inc.) software was used for data collection, 
statistical evaluation and final graph preparation. Statistical significance values and analysis-specific details are included in the result section and figure legends.

qPCR tissue expression analysis. RNA isolation, cDNA synthesis and qPCR analysis were completed in compliance with the MIQE guidelines ${ }^{57}$. Primer BLAST (http://www.ncbi.nlm.nih.gov/tools/primer-blast/) was used to develop gene-specific primers (Supplementary Table 2). Whole female, FB, thorax, MT, OVs and GT were dissected from non-blood fed, $3,12,24,48,72$ and $96 \mathrm{~h}$ post BM adult females. RNA was isolated using Trizol (Invitrogen). First-strand cDNA synthesis was performed on $1 \mu \mathrm{g}$ of total RNA in $20 \mu \mathrm{l}$ reaction using Qiagen Omniscript RT Kit (Qiagen). Transcripts were normalized by qPCR analysis of ribosomal protein S7 (rpS7) levels(49) on an Eppendorf Mastercycler ep realplex (Eppendorf) using iQ Supermix (Bio-Rad) with $25 \mu$ l volume reactions. Each experiment was done with three independent biological replicates. PCR conditions were as follows: an initial incubation at $95^{\circ} \mathrm{C}$ for $2 \mathrm{~min}$; followed by 40 cycles of $95^{\circ} \mathrm{C}$ for $15 \mathrm{~s}, 55^{\circ} \mathrm{C}$ for $15 \mathrm{~s}, 72^{\circ} \mathrm{C}$ for $20 \mathrm{~s}$.

\section{References}

1. Guedes, R. et al. Amino acids biosynthesis and nitrogen assimilation pathways: a great genomic deletion during eukaryotes evolution. BMC Genomics 12, S2 (2011).

2. Boudko, D. Y. Molecular basis of essential amino acid transport from studies of insect nutrient amino acid transporters of the SLC6 family (NAT-SLC6). J. Insect. Physiol. 58, 433-449 (2012).

3. Boudko, D. Y. In: Epithelial Transport Physiology (Springer, 2010).

4. Attardo, G. M., Hansen, I. A. \& Raikhel, A. S. Nutritional regulation of vitellogenesis in mosquitoes: implications for anautogeny. Insect. Biochem. Mol. Biol. 35, 661-675 (2005).

5. Kokoza, V. A. et al. Transcriptional regulation of the mosquito vitellogenin gene via a blood meal-triggered cascade. Gene 274, 47-65 (2001).

6. Price, D. P. et al. The fat body transcriptomes of the yellow fever mosquito Aedes aegypti, pre- and post- blood meal. PLoS ONE 6 (2011).

7. Uchida, K. et al. Induction of oogenesis in mosquitoes (Diptera: Culicidae) by infusion of the hemocoel with amino acids. J. Med. Entomol. 38, 572-575 (2001).

8. Hansen, I. A., Attardo, G. M., Park, J. H., Peng, Q. \& Raikhel, A. S. Target of rapamycin-mediated amino acid signaling in mosquito anautogeny. Proc. Natl Acad. Sci. USA 101, 10626-10631 (2004).

9. Badisco, L., Van Wielendaele, P. \& Vanden Broeck, J. Eat to reproduce: a key role for the insulin signaling pathway in adult insects. Front. Physiol. 4, 202 (2013).

10. Hansen, I. A., Attardo, G. M., Rodriguez, S. D. \& Drake, L. L. Four-way regulation of mosquito yolk protein precursor genes by juvenile hormone-, ecdysone-, nutrient-, and insulin-like peptide signaling pathways. Front. Physiol. 5, 103 (2014).

11. France, K. R. \& Judson, C. Nitrogen partitioning and blood meal utilization by Aedes aegypti (Diptera Culicidae). J. Insect. Physiol. 25, 841-846 (1979).

12. Marquardt, W. H. Biology of Disease Vectors 2nd edn. (Academic, 2004).

13. Briegel, H. Mosquito reproduction: incomplete utilization of the blood meal protein for oögenesis. J. Insect. Physiol. 31, 15-21 (1985).

14. Sappington, T. W., Hays, A. R. \& Raikhel, A. S. Mosquito Vitellogenin receptor-purification, developmental and biochemical-characterization. Insect. Biochem. Mol. Biol. 25, 807-817 (1995).

15. Sappington, T. W., Kokoza, V. A., Cho, W. L. \& Raikhel, A. S. Molecular characterization of the mosquito vitellogenin receptor reveals unexpected high homology to the Drosophila yolk protein receptor. Proc. Natl Acad. Sci. USA 93, 8934-8939 (1996).

16. Koller, C. N. \& Raikhel, A. S. Initiation of vitellogenin uptake and proteinsynthesis in the mosquito (Aedes aegypti) ovary in response to a blood meal. J. Insect. Physiol. 37, 703-711 (1991).

17. Boudko, D., Stevens, B., Donly, B. \& Harvey, W. Nutrient amino acid and neurotransmitter transporters. Compr. Mol. Insect Sci. 4, 255-309 (2005).

18. Gilbert, L. I. \& Gill, S. S. Insect Pharmacology: Channels, Receptors, Toxins and Enzymes (Academic, 2010).

19. Clements, A. N. The Biology of Mosquitoes (Chapman \& Hall, 1992).

20. Verrey, F. et al. CATs and HATs: the SLC7 family of amino acid transporters. Pflugers Arch. 447, 532-542 (2004).

21. Carpenter, V. K. et al. SLC7 amino acid transporters of the yellow fever mosquito Aedes aegypti and their role in fat body TOR signaling and reproduction. J. Insect. Physiol. 58, 513-522 (2012).

22. Attardo, G. M., Hansen, I. A., Shiao, S. H. \& Raikhel, A. S. Identification of two cationic amino acid transporters required for nutritional signaling during mosquito reproduction. J. Exp. Biol. 209, 3071-3078 (2006).

23. Hansen, I. A. et al. AaCAT1 of the yellow fever mosquito, Aedes aegypti a novel histidine-specific amino acid transporter from the slc7 family. J. Biol. Chem. 286, 10803-10813 (2011).

24. Colombani, J. et al. A nutrient sensor mechanism controls Drosophila growth. Cell 114, 739-749 (2003).

25. Bjordal, M., Arquier, N., Kniazeff, J., Pin, J. P. \& Léopold, P. Sensing of amino acids in a dopaminergic circuitry promotes rejection of an incomplete diet in Drosophila. Cell 156, 510-521 (2014).
26. Nene, V. et al. Genome sequence of Aedes aegypti, a major arbovirus vector. Science (New York, NY) 316, 1718-1723 (2007).

27. Shaffer, P. L., Goehring, A., Shankaranarayanan, A. \& Gouaux, E. Structure and mechanism of a $\mathrm{Na}+$-independent amino acid transporter. Science (New York NY) 325, 1010-1014 (2009).

28. Gao, X. et al. Mechanism of substrate recognition and transport by an amino acid antiporter. Nature 463, 828-832 (2010).

29. Eisen, L. \& Moore, C. G. Aedes (Stegomyia) aegypti in the continental United States: a vector at the cool margin of its geographic range. J. Med. Entomol. 50, 467-478 (2013).

30. Fotiadis, D., Kanai, Y. \& Palacin, M. The SLC3 and SLC7 families of amino acid transporters. Mol. Aspects Med. 34, 139-158 (2013).

31. Hundal, H. S. \& Taylor, P. M. Amino acid transceptors: gate keepers of nutrient exchange and regulators of nutrient signaling. Am. J. Physiol. Endocrinol. Metab. 296, E603-E613 (2009).

32. Closs, E. I., Boissel, J. P., Habermeier, A. \& Rotmann, A. Structure and function of cationic amino acid transporters (CATs). J. Membr. Biol. 213, 67-77 (2006)

33. Arensburger, P. et al. Sequencing of Culex quinquefasciatus establishes a platform for mosquito comparative genomics. Science (New York, NY) 330, 86-88 (2010)

34. Lawson, D. et al. VectorBase: a data resource for invertebrate vector genomics. Nucleic Acids Res. 37, D583-D587 (2009).

35. Boudko, D. Y. Bioanalytical profile of the L-arginine/nitric oxide pathway and its evaluation by capillary electrophoresis. J. Chromatogr. B. Analyt. Technol. Biomed. Life. Sci. 851, 186-210 (2007).

36. Deves, R. \& Boyd, C. A. Transporters for cationic amino acids in animal cells: discovery, structure, and function. Physiol. Rev. 78, 487-545 (1998).

37. Kim, J. W., Clos, E. I., Albritton, L. M. \& Cunningham, J. M. Transport of cationic amino acids by the mouse ecotropic retrovirus receptor. Nature 532, 725-728 (1991).

38. Hosokawa, H. Cloning and characterization of a brain-specific cationic amino acid transporter. J. Biol. Chem. 272, 8717-8722 (1997).

39. Liu, K. H. \& Tsay, Y. F. Switching between the two action modes of the dual-affinity nitrate transporter CHL 1 by phosphorylation. EMBO J. 22, 1005-1013 (2003).

40. Sun, J. Crystal structure of the plant dual-affinity nitrate transporter NRT1. Nature 507, 73-77 (2014).

41. Wang, H., Kavanaugh, M. P., North, R. A. \& Kabat, D. Cell-surface receptor for ecotropic murine retroviruses is a basic amino-acid transporter. Nature 352, 729-731 (1991).

42. Gilles, W. Monovalent cation conductance in Xenopus laevis oocytes expressing hCAT-3. Biochim. Biophys. Acta 1668, 234-239 (2005).

43. Hansen, I. A. \& Attardo, G. M. In: Short Views on Insect Molecular Biology. (ed Chandrasekar, R.) (International Book Mission, 2009).

44. Dhara, A. et al. Ovary ecdysteroidogenic hormone functions independently of the insulin receptor in the yellow fever mosquito, Aedes aegypti. Insect Biochem. Mol. Biol. 43, 1100-1108 (2013).

45. Brandon, M. C. et al. TOR signaling is required for amino acid stimulation of early trypsin protein synthesis in the midgut of Aedes aegypti mosquitoes. Insect Biochem. Mol. Biol. 38, 916-922 (2008).

46. Gulia-Nuss, M., Robertson, A. E., Brown, M. R. \& Strand, M. R. Insulin-like peptides and the target of rapamycin pathway coordinately regulate blood digestion and egg maturation in the mosquito Aedes aegypti. PLOS ONE 6 , e20401 (2011).

47. Bradley, G. L. \& Leevers, S. J. Amino acids and the humoral regulation of growth: fat bodies use slimfast. Cell 114, 656-658 (2003).

48. Luckhart, S., Vodovotz, Y., Cui, L. \& Rosenberg, R. The mosquito Anopheles stephensi limits malaria parasite development with inducible synthesis of nitric oxide. Proc. Natl Acad. Sci. USA 95, 5700-5705 (1998).

49. Hillyer, J. F. \& Estevez-Lao, T. Y. Nitric oxide is an essential component of the hemocyte-mediated mosquito immune response against bacteria. Dev. Comp. Immunol. 34, 141-149 (2010).

50. Stuehr, D. J. Enzymes of the L-arginine to nitric oxide pathway. J. Nutr. 134, 2748S-2751S discussion 2765S-2767S (2004).

51. Price, D. P., Schilkey, F. D., Ulanov, A. \& Hansen, I. A. Small mosquitoes, large implications: crowding and starvation affects gene expression and nutrient accumulation in Aedes aegypti. Parasit. Vectors 8, 252 (2015).

52. Tamura, K. et al. MEGA5: molecular evolutionary genetics analysis using maximum likelihood, evolutionary distance, and maximum parsimony methods. Mol. Biol. Evol. 28, 2731-2739 (2011).

53. Edgar, R. C. MUSCLE: multiple sequence alignment with high accuracy and high throughput. Nucleic Acids Res. 32, 1792-1797 (2004).

54. Krieger, E., Koraimann, G. \& Vriend, G. Increasing the precision of comparative models with YASARA NOVA- a self-parameterizing force field. Protiens 47, 393-402 (2002).

55. CCG Inc.. Molecular Operating Environment (MOE), 2013.08. (Chemical Computing Group, 2015). 
56. Kearse, M. et al. Geneious Basic: an integrated and extendable desktop software platform for the organization and analysis of sequence data. Bioinformatics 28, 1647-1649 (2012).

57. Huggett, J. F. et al. The digital MIQE guidelines: minimum information for publication of quantitative digital PCR experiments. Clin. Chem. 59, 892-902 (2013).

\section{Acknowledgements}

This work was supported by grant \# 1SC1AI109055 from the National Institute of Health.

\section{Author contributions}

D.Y.B., H.T. and I.A.H. designed the research; D.Y.B., S.D.R, E.A.M., H.T. and L.L.D. performed the research; D.Y.B., H.T., D.P.P., L.L.D. and I.A.H. analyzed the data; D.Y.B., H.T. and I.A.H. wrote the paper. D.Y.B. and H.T. contributed equally to this work.

\section{Additional information}

Supplementary Information accompanies this paper at http://www.nature.com/ naturecommunications

Competing financial interests: The authors declare no competing financial interests.

Reprints and permission information is available online at http://npg.nature.com/ reprintsandpermissions/

How to cite this article: Boudko, D. Y. et al. Substrate specificity and transport mechanism of amino acid transceptor Slimfast from Aedes aegypti. Nat. Commun. 6:8546 doi: $10.1038 /$ ncomms9546 (2015).

\section{(c) (i)} International License. The images or other third party material in this article are included in the article's Creative Commons license, unless indicated otherwise in the credit line; if the material is not included under the Creative Commons license, users will need to obtain permission from the license holder to reproduce the material To view a copy of this license, visit http://creativecommons.org/licenses/by/4.0/ 